\title{
Correlação entre independência funcional e risco de quedas em idosos de três instituições de longa permanência*
}

\author{
Correlation between functional independence and risk of falls in older adults at three \\ long-term care facilities \\ Correlación entre la independencia funcional y el riesgo de caídas en los ancianos en tres \\ instituciones de larga permanencia
}

Como citar este artigo:

Paula JGF, Gonçalves LHT, Nogueira LMV, Delage PEGA. Correlation between functional independence and risk of falls in older adults at three long-term care facilities. Rev Esc Enferm USP. 2020;54:e3601. doi: https://doi.org/10.1590/S1980-220X2018054103601

\section{Jully Greyce Freitas de Paula ${ }^{1}$ \\ D Lucia Hisako Takase Gonçalves ${ }^{2}$ \\ Daura Maria Vidal Nogueira ${ }^{1}$ \\ Paulo Elias Gotardelo Audebert Delage $^{1}$}

* Extraído da dissertação: "Análise da correlação
entre a independência funcional e o risco
de quedas em idosos institucionalizados",
Universidade do Estado do Pará, 2018.
${ }^{1}$ Universidade do Estado do Pará, Belém,
PA, Brasil.
${ }^{2}$ Universidade Federal do Pará, Belém, PA, Brasil.

\begin{abstract}
Objective: To verify the correlation between functional independence and the risk of falls in a group of institutionalized older adults. Method: A cross-sectional, observational, quantitative study conducted in three Long-Term Care Facilities for older adults in the Municipality of Belém, involving both genders and assessed using the Katz Index and Tinetti Index. Pearson's Chi-squared test was used for analysis, adopting a p-value significance level of $<0.05$. The data correlation was performed using the Pearson correlation test with a significance of $5 \%(\mathrm{p}<0.05)$. Results: Forty-eight $(48)$ older adults participated.Most were classified as functionally independent (89.6\%) and with a low risk of falls (58.3\%). There was a moderate correlation between functional independence and low risk of falls. Conclusion: The more independent the older adult is, the lower the risk of falls. Therefore, it is necessary to plan individualized care, considering its peculiarities and limitations, so that older adults can preserve their functional independence for longer.
\end{abstract}

DESCRIPTORS

Aged; Homes for the Aged; Accidental Falls; Activities of Daily Living; Geriatric Nursing. 


\section{INTRODUÇÃO}

O termo independência funcional está relacionado à mobilidade e capacidade funcional que permite ao indivíduo realizar Atividades da Vida Diária (AVD) com independência, indicando condições motoras e cognitivas adequadas para a execução das mesmas ${ }^{(1)}$. A limitação para desenvolver tais atividades pode comprometer o dia a dia das pessoas, sobretudo de idosos, com implicação em risco de quedas, podendo ocasionar prejuízos irreparáveis à saúde, gerando incapacidades e até mesmo a morte ${ }^{(2)}$.

Anualmente, aproximadamente de $30 \%$ a $50 \%$ dos idosos institucionalizados sofrem quedas e $40 \%$ deles experimentam quedas recorrentes ${ }^{(3)}$. As quedas estão entre as principais causas de trauma entre idosos, sendo consideradas a sexta causa de óbito por lesões acidentais e não acidentais ${ }^{(4)}$. Após cair, o idoso pode sofrer várias consequências, como ferimentos graves, escoriações, arranhões e fraturas, o que pode contribuir para o declínio funcional na realização das AVD, isolamento social e maior vulnerabilidade para recorrência de quedas ${ }^{(5)}$.

De acordo com a Agência Nacional de Vigilância Sanitária (ANVISA), uma Instituição de Longa Permanência para Idosos (ILPI) é uma entidade governamental ou não-governamental destinada a acolher pessoas com 60 anos ou mais, com ou sem suporte familiar, garantindo a dignidade, liberdade e cidadania dos idosos ${ }^{(6)}$.

Ao ser institucionalizado, o idoso enfrenta uma série de limitações em sua rotina, podendo experimentar uma redução em sua autonomia, associada a um maior sedentarismo e sentimento de perda e abandono. Este quadro pode muitas vezes levar a uma progressiva perda da independência funcional e consequente aumento do risco de quedas ${ }^{(7)}$. Assim, é essencial que o profissional de enfermagem leve em consideração os fatores físico-ambientais e biopsicossociais no momento de traçar o plano de cuidados, de modo a garantir que os idosos possam realizar suas AVD sem riscos de sofrerem lesões por quedas ${ }^{(8)}$. Diante disto, o objetivo desta pesquisa foi avaliar de que modo a independência funcional de um grupo de idosos institucionalizados se relaciona com seus riscos de quedas.

\section{MÉTODO}

\section{TIPO DE ESTUDO}

Estudo transversal, observacional, de abordagem quantitativa.

\section{Cenário}

O estudo foi realizado em três ILPIs no Município de Belém, sendo uma instituição governamental mantida pelo Estado e as outras duas filantrópicas. Neste estudo, as instituições foram identificadas pelas letras A, B e C. As três instituições abrigam um total de 120 idosos.

Atualmente, a instituição A abriga 44 idosos, sendo 19 homens e 25 mulheres, na faixa etária de 60 a 100 anos, com os três graus de dependência funcional. A instituição B é filantrópica e é exclusiva para mulheres, a qual abriga 24 idosas com faixa etária de 60 a 99 anos, com grau de dependência I e grau de dependência II. Por fim, a instituição C é uma entidade filantrópica e abriga 79 idosos com os três graus de dependência funcional, sendo 11 homens e 68 mulheres.

\section{Critérios de SELEÇão}

Os critérios de inclusão foram: pessoas com idade igual ou superior a 60 anos, residentes na ILPI pelo tempo mínimo de 12 meses e lúcidos, com capacidade para responder às questões físicas e funcionais. Foram excluídos os idosos que apresentavam incapacidade funcional (restrito ao leito e usuários de cadeira de rodas).

Para o cálculo amostral foi utilizado o número total de idosos $(\mathrm{N}=120)$ que residiam nos locais do estudo. Essa população de referência foi obtida segundo informações das próprias instituições após o cálculo amostral $\left(n=N \times n_{0} /\left(N+n_{0}\right) \text {, onde } n_{0}=1 / E_{0}^{2} \text { e } E_{0} \text { é o erro amostral }\right)^{(9)}$. Considerando-se valor alfa de 0,05 , obteve-se amostra inicial estimada de $n=46$ idosos, que foi atendida, uma vez que participaram do estudo 48 idosos.

\section{Coleta De Dados}

A coleta de dados ocorreu no período de janeiro a março de 2018. Inicialmente os idosos responderam a um instrumento elaborado pelos próprios pesquisadores, dividido em três partes: dados sociodemográficos, de saúde e sobre o risco de quedas. Além disso, a Circunferência da Panturrilha (CP) também foi mensurada pelos pesquisadores para a identificação de sarcopenia, com base no valor de referência proposto pelo Consenso Europeu para Definição Diagnóstica da Sarcopenia ${ }^{(10)}$. A CP foi mensurada com uma fita métrica bilateralmente, contornando a maior curvatura da panturrilha. Considerou-se o valor normal de $\geq 31 \mathrm{~cm}$. As medidas abaixo desse valor foram consideradas indicativos de sarcopenia.

As variáveis independentes do estudo foram divididas em características sociodemográficas, características clínicas e situação de exposição a quedas. As características sociodemográficas são: idade, gênero, procedência, estado civil, número de filhos, escolaridade, iniciativa de morar e o tempo de residência na instituição. As características clínicas são: comorbidades, estado de visão autorreferida e circunferência da panturrilha. A situação de exposição a quedas foi levantada com base nas seguintes informações: histórico de quedas, uso de auxílio para se movimentar, avalição do risco de quedas e avaliação ambiental. Por fim, a variável-desfecho foi a "independência funcional quanto ao risco de quedas" conforme o escore do Índice de Katz e Índice de Tinetti ${ }^{(11)}$.

Para conhecer o grau de independência funcional em atividade de vida diária foi aplicado o Índice de $\mathrm{Katz}^{(11)}$, a partir da observação da execução das AVD. A escala consta de seis itens que mensuram a capacidade de realizar ações relacionadas ao autocuidado, a saber: tomar banho, vestir-se, ir ao banheiro, transferência, continência e alimentação ${ }^{(11)}$. 
Após a avaliação, foi realizada a classificação nas categorias conforme sugerido por Katz: independentes, dependência moderada e muito dependentes ${ }^{(11)}$. Foram considerados independentes aqueles que alcançaram escore entre cinco e seis pontos; com dependência moderada os que tiveram de três a quatro pontos; e como muito dependentes os que tiveram de um a dois pontos. Esta classificação foi obtida a partir da soma dos valores relativos a cada critério. Neste caso, o escore zero era indicativo de dependência e necessidade de supervisão para realização de suas atividades e o escore um correspondia à independência na realização dessas atividades.

Para a avaliação do risco de quedas foi utilizado o Índice de Tinetti ${ }^{(11)}$, que permite avaliar o equilíbrio e a marcha do idoso. Esta escala apresenta 16 itens, cada um pontuável entre 0 a 1 ou de 0 a 2 . Zero indica o nível máximo de incapacidade, enquanto 1 ou 2 correspondem a graus variados de incapacidade, sendo que, dependendo do exercício avaliado, a nota máxima pode ser 1 ou 2 . A escala avalia o equilíbrio, que possui a pontuação máxima de 16; e a marcha, com a pontuação máxima de 12 . A soma dessas avaliações pode chegar no máximo a 28 pontos, e a partir da pontuação total da avaliação, pode ser classificada em baixo ( 25 a 28 pontos), médio (19 a 24 pontos) ou elevado risco de quedas (inferior a 19 pontos) $)^{(12)}$.

Os testes de Índice de Tinetti ${ }^{(11)}$ e Índice de Katz ${ }^{(11)}$ foram realizados com o auxílio de uma aluna da graduação de enfermagem que foi treinada para a execução desses testes. Esta avaliação foi realizada nos pátios recreativos das instituições que tinham boa luminosidade e o solo plano e regular.

Para verificar as condições ambientais das instituições em que os idosos residem, por ocasião da coleta de dados, foi aplicado pela equipe de pesquisa um check-list recomendado pelo Ministério da Saúde ${ }^{(13)}$, observando aspectos como área de locomoção, iluminação, piso, escada, entre outros. A pontuação final desse instrumento é dada por cada resposta negativa, que vale um ponto. A pontuação final é a soma de todos os itens da escala, sendo que a pontuação máxima é de 14 pontos. Quanto maior a pontuação, maior o risco ambiental para a ocorrência de quedas. Esta avaliação ambiental foi feita por meio da observação dos cômodos da instituição.

\section{AnÁlise e TRATAMENTO DOS DADOS}

Foi utilizado o teste não paramétrico Qui-quadrado de Pearson para tendência/aderência entre variáveis nominais, adotando-se nível de significância de $\mathrm{p}$-valor $<0,05$. O teste de correlação de Pearson foi utilizado para verificar a correlação entre a independência funcional dos idosos e o risco de quedas, com nível de $95 \%$ de confiança e 5\% de significância. Para o teste de Pearson ter significado estatístico, o valor de coeficiente de correlação $(r)$ deve ser julgado considerando o tamanho da amostra $(n)^{(14)}$. Considera-se que o valor de $r$ é: $0<\mathrm{r}<0,25$ ou $-0,25<r<0$ : correlação pequena ou nula; $0,25<r<0,50$ ou $-0,50<r<-0,25$ : correlação fraca; $0,50<r<0,75$ ou $-0,75<r<-0,50$ : correlação moderada; $0,75<r<1,00$ ou $-1,00<r<-0,75$ : correlação forte ou perfeita (perfeita se $r=-1$ ou $r=1$ ).

Os dados foram analisados por meio da estatística descritiva e inferencial, fazendo-se uso do Microsoft Excel e do Statistical Package for the Social Sciences (SPSS) versão 24.0, todos em ambiente Windows 7.

\section{AsPeCtos ÉTICOS}

Esta pesquisa foi desenvolvida de acordo com as diretrizes e normas regulamentadoras de pesquisas envolvendo seres humanos, expressos através da Resolução no 466 de 12/12/2012, do Conselho Nacional de Saúde. Foi aprovada pelo Comitê de Ética (CEP) da Universidade do Estado do Pará, conforme o Parecer no 2.700.091, em 07/06/2018.

\section{RESULTADOS}

Foram inseridos no estudo 48 participantes, com média de idade de 76,5 $( \pm 10,2)$ anos, sendo que $72,9 \%$ eram mulheres e $27,1 \%$ eram homens. Houve o predomínio de solteiros $(58,3 \%)$, seguido dos que eram viúvos $(27,1 \%)$, sendo que $56,3 \%$ dos idosos não tinham filhos. A maioria dos participantes da pesquisa possuíam baixa escolaridade, sendo que $22,9 \%$ nunca estudaram e $45,8 \%$ tiveram o tempo de estudo entre um e cinco anos. Verificou-se que 52,1\% dos idosos foram residir na instituição por iniciativa própria, e o tempo de institucionalização da maioria dos idosos variou entre um e cinco anos $(60,4 \%)$.

Com relação a existência de enfermidade, $81,3 \%$ dos idosos relataram sofrer algum tipo de morbidade, sendo a hipertensão arterial $(50,0 \%)$ a de maior prevalência, seguida por diabetes mellitus (25,0\%). Quanto ao estado da visão autorreferida, 37,5\% consideraram a visão como boa e $25,0 \%$ como ótima. Entretanto, verificou-se que $33,3 \%$ consideram a sua visão entre ruim e péssima, sendo que destes, 10,4\% declararam ter muita dificuldade em realizar $\mathrm{AVD}$.

Quanto a CP, observou-se que 83,3\% dos idosos possuem a circunferência da panturrilha maior ou igual a $31 \mathrm{~cm}$. Verificou-se que os $16,7 \%$ dos idosos que possuíam a $\mathrm{CP}$ menor que $31 \mathrm{~cm}$ eram do sexo feminino, o que corresponde a 22,9\% de todas as mulheres participantes do estudo.

Verificou-se que $45,8 \%$ dos idosos já sofreram queda no último ano, sendo que destes, $39,6 \%$ declararam ter sofrido de uma a quatro quedas, e 6,3\% sofreram mais de cinco quedas. Dentre os motivos relatados pelos idosos, 45,5\% afirmaram que o desequilíbrio foi o principal motivo de quedas e que os locais com maior frequência de quedas foram o banheiro $(31,8 \%)$ e o quarto $(31,8 \%)$. Apenas 35,4\% dos idosos afirmaram precisar de auxílio para locomoção, sendo a bengala $(64,7 \%)$ o principal auxílio utilizado. A Tabela 1 mostra a classificação de quedas de acordo com a avaliação realizada por meio do Índice de Tinetti ${ }^{(11)}$. Nesse caso, a maioria dos idosos foi classificada com baixo risco de quedas $(58,3 \%)$, sendo esta uma tendência significativa $(\mathrm{p}<0,05)$. 
Tabela 1 - Classificação do risco de quedas de acordo com o Índice de Tinetti em idosos institucionalizados - Belém, PA, Brasil, 2018.

\begin{tabular}{|c|c|c|c|}
\hline Classificação do risco de quedas (Índice de Tinetti) & $N$ & $\%$ & p-valor ${ }^{*}$ \\
\hline Baixo risco de quedas & 28 & 58,3 & \\
\hline Médio risco de quedas & 9 & 18,8 & $<0,0001$ \\
\hline Alto risco de quedas & 11 & 22,9 & \\
\hline
\end{tabular}

(*) Teste Qui-quadrado de Pearson para tendência (p-valor $<0,05)$. Nota: $(\mathrm{N}=48)$

Sobre a avaliação ambiental do risco de quedas, $31,58 \%$ dos idosos convivem com revestimentos não uniformes ou tapetes não fixos nas áreas de locomoção e 17,9\% dos idosos convivem com áreas de locomoção sem barras de apoio nas ILPIs. Observou-se que $17,9 \%$ dos idosos convivem com iluminação insuficiente para clarear totalmente o interior de cada cômodo, incluindo degraus. No banheiro, 13,6\% dos idosos reportaram que a área do chuveiro não possui piso antiderrapante. Além disso, 19,0\% dos idosos vivem em uma instituição em que o piso da escada não possui revestimento antiderrapante.

A Tabela 2 mostra a classificação da dependência funcional de acordo com a avaliação realizada utilizando o Índice de Katz ${ }^{(11)}$. A maioria dos idosos foi classificada como independente funcionalmente $(89,6 \%)$, sendo esta uma tendência significativa $(\mathrm{p}<0,05)$.

Tabela 2 - Classificação da dependência funcional de acordo com o Índice de Katz em idosos institucionalizados - Belém, PA, Brasil, 2018.

\begin{tabular}{lccc}
\hline Índice Katz & N & \% & p-valor ${ }^{(*)}$ \\
\hline Independente & 43 & 89,6 \\
Dependência moderada & 4 & 8,3 & $<0,0001$ \\
Muito dependente & 1 & 2,1 & \\
\hline
\end{tabular}

${ }^{(*)}$ Teste Qui-quadrado de Pearson para tendência (p-valor $\left.<0,05\right)$.

Nota: $(n=48)$

Ao correlacionar a independência funcional para as atividades de vida diária (Índice de Katz) e o risco de quedas em idosos institucionalizados (Índice de Tinetti), o resultado deste coeficiente foi de 0,602 , que indica uma correlação moderada, considerando $\mathrm{p}$-valor de $<0,05$. Este resultado pode ser demonstrado por meio da Figura 1.

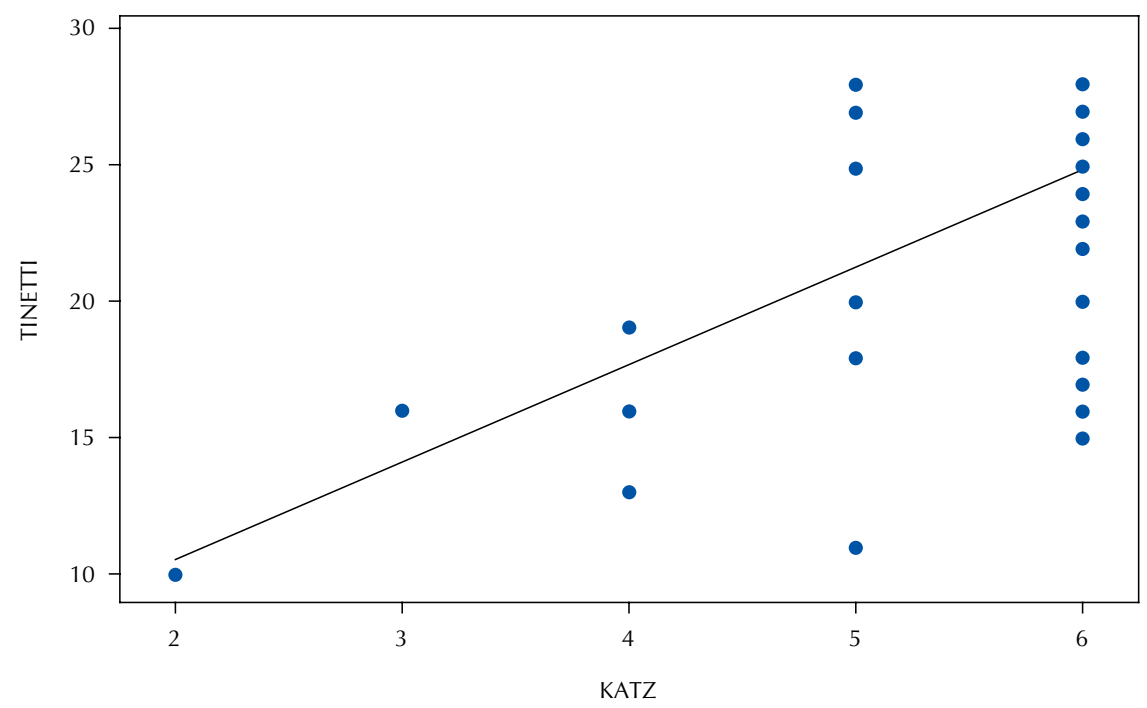

Figura 1 - Teste de Correlação de Pearson entre a independência funcional para as atividades de vida diária e o risco de quedas em idosos institucionalizados. 
O teste de correlação de Pearson aponta que há correlação positiva moderada $(\mathrm{r}=0,602)$ e significativa $(\mathrm{p}<0,05)$ entre o escore obtido pelos idosos no Índice de Katz ${ }^{(11)}$, que mede o grau de independência funcional, e no instrumento Tinetti, que aponta o risco de quedas entre os idosos avaliados. O elevado escore obtido nos dois instrumentos remete a evidências de que quanto maior a independência funcional, menor será o risco de quedas.

\section{DISCUSSÃO}

No presente estudo, a média de idade dos participantes foi similar a outro estudo com idosos institucionalizados ${ }^{(15)}$. A maioria dos participantes do estudo foram mulheres, corroborando outros estudos com idosos institucionalizados em que, também, predominaram participantes do sexo feminino ${ }^{(15-16)}$. Este fenômeno pode ser explicado pela maior sobrevida das mulheres, pela maior probabilidade de elas terem as piores condições de saúde e de capacidade funcional, além de uma posição desfavorável nas relações familiares, já que os homens têm maior chance de serem cuidados por seus cônjuges, permanecendo, dessa forma, mais tempo com a família ${ }^{(17)}$.

Quanto a situação conjugal, a maioria era solteiro, seguidos pelos que eram viúvos, corroborando os dados de um estudo similar ${ }^{(15,17)}$. A ausência de um parceiro e o apoio social enfraquecido são fatores que podem levar a institucionalização do idoso ${ }^{(16)}$.

A maioria dos idosos não possui filhos. $O$ fato de não possuir filhos é muitas vezes descrito como uma predisposição a ser institucionalizado. Sendo assim, é possível identificar uma vulnerabilidade no saber cuidar do idoso por parte da família, que pode ser observada quando a responsabilidade do cuidado é direcionada para cuidadores não familiares devido a diminuição da independência e da capacidade funcional ${ }^{(16)}$.

Constatou-se uma baixa escolaridade dos idosos avaliados neste estudo. Esta situação é reflexo de uma herança de décadas passadas, quando o trabalho braçal e o doméstico eram mais valorizados do que a formação intelectual ${ }^{(18)}$.

Além disso, verificou-se que a maioria dos participantes da pesquisa foi residir na instituição por iniciativa própria, contrariando os dados de outro estudo ${ }^{(19)}$, onde todos os idosos declararam que residiam ali por não terem outra opção. A literatura aponta que dentre os motivos associados à institucionalização do idoso está o fato de não possuírem cuidador, conflitos familiares, abandono, "estar doente" e não ter lugar para morar ${ }^{(20)}$. A maioria dos idosos reside na ILPI, no período de um a cinco anos. $\mathrm{O}$ tempo de permanência e senso de pertencimento àquele lugar é relevante, uma vez que o lar não compreende apenas um espaço físico, mas também representa um local que faz parte da vida dos moradores, como as histórias vividas, recordações, um local para o descanso e de proteção ${ }^{(20)}$.

Quanto às condições de saúde dos idosos estudados, observou-se que a maioria declarou a existência de alguma enfermidade, sendo que a doença crônica de maior prevalência foi a hipertensão arterial sistêmica. Este achado corrobora outra pesquisa realizada em ILPI $^{(18)}$.
Com relação ao estado da visão, observou-se que alguns idosos indicaram possuir visão entre "ruim" e "péssima”. É importante deixar claro que não foi realizada qualquer avaliação oftalmológica na ocasião da pesquisa. Esses dados foram baseados no autorelato do estado de visão pelos próprios participantes. Quando a visão está comprometida, ela pode contribuir com a perda do equilíbrio, visto que a visão auxilia no controle postural. Além disso, uma baixa luminosidade ambiental e infraestrutura inadequada podem acentuar o risco de quedas ${ }^{(7,21)}$.

Foi identificado que 22,9\% das participantes, todas do sexo feminino, tinham a CP abaixo de $31 \mathrm{~cm}$, o que pode ser considerando um indicativo de sarcopenia. A literatura aponta que esta patologia é predominante em mulheres ${ }^{(22)}$ e que sua ocorrência pode estar associada ao baixo peso, presença de doenças crônicas, à limitações e à incapacidade funcional ${ }^{(23)}$.

Apesar dos dados não mostrarem uma alta prevalência de participantes com $\mathrm{CP}<31$, é preciso ter maior atenção aos idosos obesos, que podem apresentar a $\mathrm{CP}$ normal ou aumentada, mesmo na presença de sarcopenia, podendo ser indicativo de obesidade sarcopênica. Porém, neste estudo não foi verificado o Índice de Massa Corporal (IMC) para avaliação do estado nutricional.

Com relação aos dados sobre a ocorrência de quedas, verificou-se que $45,8 \%$ dos idosos sofreram quedas, assemelhando-se ao resultado de um estudo realizado com idosos institucionalizados em Uberlândia/ $\mathrm{MG}^{(20)}$, em que 42,8\% dos participantes relataram ter caído. Destes idosos que caíram, identificou-se que as mulheres caíram mais do que os homens, corroborando dados presentes na literatura ${ }^{(23)}$ que encontraram $71,42 \%$ da ocorrência de quedas em mulheres. Segundo os autores, uma das justificativas que pode contribuir para este evento é a maior incidência de doenças crônicas degenerativas entre as idosas.

Em ILPIs, é importante destacar a presença do "idoso caidor", que é aquele que apresentou mais de duas quedas no último ano ${ }^{(24)}$. Esse evento é mais frequente em idosos institucionalizados devido a sua maior fragilidade e dependência funcional, pois estão mais expostos a doenças e ao uso de maior quantidade de medicamentos, o que pode contribuir para o maior risco de quedas ${ }^{(25)}$. Levando em consideração o "idoso caidor", vale destacar a importância de verificar aspectos relacionados com a alteração de humor, como a presença da ansiedade, depressão e medo de cair, fatores que podem trazer implicações para a mobilidade, tornando-a restrita, além de contribuir para novas quedas ${ }^{(26)}$.

Dentre os motivos de quedas, identificou-se que o desequilíbrio foi o mais prevalente, seguido pelo motivo de escorregar. O déficit de equilíbrio está presente em indivíduos que já caíram, pois apresentam maior oscilação ântero-posterior em posição ereta comparado àqueles que nunca caíram ${ }^{(27)}$.

De acordo com o relato dos idosos sobre o local de ocorrência de queda, a maioria caiu no banheiro e no quarto. Sendo assim, é de suma importância a realização do monitoramento destes locais pela equipe de saúde ${ }^{(21)}$, que deve receber capacitação de prevenção de quedas visando a qualidade da assistência e segurança dos idosos. Com relação a 
necessidade de utilização de dispositivo de auxílio de locomoção entre os idosos, verificou-se que dentre aqueles que precisam de algum dispositivo auxiliar de marcha, a bengala é o principal dispositivo de auxílio utilizado, corroborando dados de um estudo anterior em que esta era utilizada por $10 \%$ dos idosos ${ }^{(28)}$.

Identificou-se que em algumas das ILPIs utilizadas na pesquisa, a presença de pisos não uniformes pode contribuir para a ocorrência de quedas. Segundo estudos, é comum ocorrer quedas em pisos irregulares, mesmo que não estejam molhados ${ }^{(18)}$. Outro fator ambiental encontrado em uma das ILPI foi a falta de barras de apoio nas áreas de locomoção. A Agência Nacional de Vigilância Sanitária (ANVISA) recomenda que as áreas de circulações com largura maior ou igual a $1,50 \mathrm{~m}$ devem possuir corrimão dos dois lados; e as áreas de circulações com largura menor que $1,50 \mathrm{~m}$ podem possuir corrimão em apenas um dos $\operatorname{lados}^{(4)}$.

Quanto a iluminação, verificou-se em uma ILPI do presente estudo que, durante o dia, em alguns cômodos, como em áreas de circulação e quartos, a iluminação era insuficiente, dificultando a visualização de degraus ou objetos que estivessem no caminho. A utilização da luz artificial poderia complementar a segurança dos idosos residentes. A RDC No $283 / 2005^{(4)}$ recomenda que as áreas de circulação internas devem ter luz de vigília permanente, assim como os dormitórios e refeitórios devem ser dotados de luz de vigília.

Em duas ILPIs desta pesquisa, os banheiros não possuíam piso antiderrapante nas áreas do chuveiro. Inclusive, esta foi uma queixa pertinente entre os idosos. De acordo com a RDC 283/2005(4), o banheiro não deve ter desnível em forma de degrau e nem revestimentos que produzam brilhos e reflexos.

De acordo com a avaliação da independência funcional por meio do Índice de $\mathrm{Katz}^{(11)}$, verificou-se que a maioria dos idosos foram classificados como independentes funcionalmente. Este resultado foi o contrário do estudo realizado em ILPIs de Uberlândia, pois a proporção de idosos independentes em suas AVD foi de $33 \% \%^{(20)}$.

Quanto a avaliação do risco de quedas, notou-se que a maioria possuía baixo risco de quedas, corroborando o resultado de outro estudo ${ }^{(29)}$. Além disso, todos os idosos que possuíam alto risco de quedas eram pertencentes ao sexo feminino. Apesar destes dados reforçarem a literatura e serem compatíveis com particularidades da fisiologia e do processo de envelhecimento feminino ${ }^{(30)}$, é preciso levar em consideração a disparidade de participantes do sexo feminino na amostra deste estudo, com uma proporção quase três vezes maior em relação ao público masculino.

Constatou-se nesta pesquisa que há uma correlação positiva moderada entre a independência funcional e o risco de quedas, ou seja, quanto mais independentes são os idosos, menor o risco de quedas. Verificou-se, ainda, que a maioria dos idosos foram considerados independentes. Vale ressaltar que o fato de serem independentes não isenta os idosos de sofrerem queda ${ }^{(30)}$. Por exemplo, no presente estudo $44,2 \%$ dos idosos independentes relataram que sofreram pelo menos uma queda no último ano.
Uma queda anterior não parece ser suficiente para causar uma nova queda, mas sim quando está associada à interação entre as habilidades dos idosos, os fatores intrínsecos (presença de doenças crônicas, declínio funcional, entre outros) e o grau de exposição à fatores de risco extrínsecos. A combinação desses fatores pode ter contribuído para resultar em uma correlação moderada entre a independência e o risco de quedas.

Sendo assim, a manutenção da autonomia e da independência é importante para diminuir as dificuldades em realizar as AVD, algo que é positivo tanto para os próprios indivíduos quanto para a família e a sociedade ${ }^{(30)}$. Vale destacar que, para isso, torna-se imprescindível, por parte dos profissionais que acompanham os idosos institucionalizados, o monitoramento dos fatores de risco de forma individualizada para a prevenção deste evento. Para a enfermagem, principalmente na área gerontogeriátrica, é necessário se apropriar melhor dos recursos e instrumentos que auxiliam na identificação do nível funcional do idoso a fim de elaborar melhor as estratégias e ações de promoção de saúde, de modo a contribuir para o envelhecimento mais saudável, com maior autonomia e independência.

O presente estudo apresenta algumas limitações. Em primeiro lugar, o delineamento transversal aqui adotado implica na impossibilidade de monitorar a sequência temporal entre os fatores de risco (exposição) e a independência funcional e risco de quedas (desfecho), os quais devem ser monitorados/ avaliados constantemente. Portanto, sugere-se que novos estudos sejam realizados com outros delineamentos para avaliar mais detalhadamente esse fenômeno.

Outra limitação diz respeito a um certo viés de amostra no que concerne ao perfil dos participantes, mais particularmente relacionado ao sexo, pois uma das instituições em que foi realizado o estudo era exclusivamente feminina. Também houve limitação na identificação de idosos que apresentassem obesidade sarcopênica, pois não foram realizadas medidas de outros preditores de sarcopenia: IMC, força (preensão manual) e performance física (velocidade da marcha). Sugere-se para estudos futuros a realização de outras medidas e a utilização de critérios para a identificação de sarcopenia.

Apesar das limitações identificadas, os autores consideram que os resultados deste estudo podem contribuir para compor a produção científica sobre funcionalidade e risco de quedas em idosos institucionalizados no contexto do estado do Pará.

\section{CONCLUSÃO}

A correlação entre independência funcional e o risco de quedas foi moderada, indicando que quanto mais independentes são os idosos, menor o risco de quedas. Diante desta constatação, é necessário fazer um planejamento de cuidados individualizados considerando suas peculiaridades e limitações, de modo que o idoso possa preservar por mais tempo sua independência funcional.

Para a prevenção de quedas, sugere-se que haja um maior monitoramento nas ILPI em relação aos idosos que já sofreram alguma queda, já que esse evento é um preditor de novas 
quedas. Portanto, recomenda-se às ILPIs a adequação da estrutura do ambiente de modo a torná-lo seguro, já que neste estudo, os lugares que tiveram maior registro de quedas foram os quartos e banheiros. Além disso, ressalta-se a importância da educação continuada para os profissionais de saúde e cuidadores, de forma a capacitá-los na identificação de situações que coloquem os idosos em risco de quedas. Por fim, sugere-se ainda a realização de educação em saúde com os idosos sobre os riscos de queda, prevenção, importância do uso de dispositivos de auxílio para locomoção e realização de atividade física.

Os resultados encontrados neste estudo fornecem subsídios orientadores para o planejamento de intervenções de enfermagem voltadas para preservação da capacidade funcional, com vistas a promover a segurança dos idosos institucionalizados.

\section{RESUMO}

Objetivo: Verificar a correlação entre a independência funcional e o risco de quedas em um grupo de idosos institucionalizados. Método: Estudo transversal, observacional, quantitativo, realizado em três Instituições de Longa Permanência para Idosos no Município de Belém, de ambos os gêneros, avaliados por meio do Índice de Katz e Índice de Tinetti. Para análise utilizou-se o teste de Qui-quadrado de Pearson, adotando-se um nível de significância de p-valor < 0.05. E a correlação dos dados foi realizada pelo teste de correlação de Pearson, com significância de 5\% $(p<0,05)$. Resultados: Participaram 48 idosos. A maioria foi classificada como funcionalmente independente $(89,6 \%)$ e com baixo risco de quedas $(58,3 \%)$. Houve correlação moderada entre a independência funcional e baixo riscos de quedas. Conclusão: Quanto mais independentes são os idosos, menor é o risco de quedas. Portanto, é necessário fazer um planejamento de cuidados individualizados, considerando suas peculiaridades e limitações, de modo que o idoso possa preservar por mais tempo sua independência funcional.

\section{DESCRITORES}

Idoso; Instituição de Longa Permanência para Idosos; Acidentes por Quedas; Atividades Cotidianas; Enfermagem Geriátrica.

\section{RESUMEN}

Objetivo: Comprobar la correlación entre la independencia funcional y el riesgo de caídas en un grupo de ancianos institucionalizados. Método: Estudio transversal, observacional, cuantitativo, realizado en tres Instituciones de Larga Permanencia para Ancianos en el municipio de Belém, de los dos géneros, evaluados por medio del Índice de Katz y del Índice de Tinetti. Para análisis fue utilizado el test de Qui-quadrado de Pearson, adoptando un nivel de significado de $\mathrm{p}$-valor $<0.05$. Y la correlación de los datos fue realizada por el test de correlación de Pearson, con significado de 5\% $(p<0,05)$. Resultados: Participaron 48 ancianos. La mayoría fue clasificada como funcionalmente independiente $(89,6 \%)$ y con bajo riesgo de caídas $(58,3 \%)$. Hube correlación moderada entre la independencia funcional y bajo riesgo de caídas. Conclusión: Cuanto más independientes son los ancianos, menor es lo riesgo de caídas. Entonces, es necesario hacer um planeamiento de cuidados individualizados, considerando sus peculiaridades y limitaciones, para que el anciano pueda preservar por más tiempo su independencia funcional.

\section{DESCRIPTORES}

Anciano; Hogares para Ancianos; Accidentes por Caídas; Actividades Cotidianas; Enfermería Geriátrica.

\section{REFERÊNCIAS}

1. Ferraresi JR, Prata MG, Scheicher ME. Avaliação do equilíbrio e do nível de independência funcional de idosos da comunidade. Rev Bras Geriatr Gerontol [Internet]. 2015 [citado 2018 maio 27];18(3):499-506. Disponível em: http://www.scielo.br/scielo.php?pid=s180998232015000300499\&script=sci_abstract\&tlng=pt

2. Sarges NA, Santos MIPO, Chaves EC. Evaluation of the safety of hospitalized older adults as for the risk of falls. Rev Bras Enferm [Internet]. 2017 [citado 2018 dez. 29];70(4):860-7. Disponível em: http://www.scielo.br/scielo.php?script=sci_arttext\&pid=S0034$71672017000400860 \& \operatorname{lng}=\mathrm{en}$

3. Organização Mundial da Saúde. Relatório global da OMS sobre prevenção de quedas na velhice [internet]. São Paulo: Secretaria de Estado da Saúde; 2010 [citado 2016 maio 10]. Disponível em: http://www.saude.sp.gov.br/resources/ccd/publicacoes/publicacoes-ccd/ saude-e-populacao/manual_oms_-_site.pdf

4. Tinetti ME. Factors associated with serious injury during falls by ambulatory nursing home residents. J Am Geriatr Soc. 1987;35(7):644-8.

5. Alves A, Araújo-Patrício A, Fernandes K, Duarte M, Santos J, Oliveira M. Ocorrências de quedas entre idosos institucionalizados: prevalência, causas e consequências. Rev Online Pesqui Cuid Fundam [Internet]. 2016 Apr 4; [Cited 2017 Jun 13];8(2):4376-4386. Available from: http://www.seer.unirio.br/index.php/cuidadofundamental/article/view/4438/pdf_1885

6. Brasil. Ministério da Saúde; Agência Nacional de Vigilância Sanitária. Resolução RDC n. 283, de 26 de setembro de 2005. Aprova o regulamento Técnico que define normas de funcionamento para as instituições de longa permanência para idosos [Internet]. Brasília; 2005 [citado 2018 dez. 15]. Disponível em: http://portal.anvisa.gov.br/documents/10181/2718376/RDC_283_2005_COMP.pdf

7. Baixinho CRSL, Dixe MACR. Falls in institutions for older adults: characterization of fall occurrences and associated risk factors. Rev Eletr Enferm [internet]. 2015 [cited 2018 Jul. 17];17(4):1-9. Available form: https://revistas.ufg.br/fen/article/view/31858/20666

8. Oliveira FS, Santos SSC, Kerber NPC; Francioni FF; Cruz VDC. Produção científica acerca dos fatores de risco ambientais para quedas em idosos: revisão integrativa. Rev Enferm UFPE [internet]. 2015 [citado 2018 jul. 12];9(2):759-67. Disponível em:https://periodicos.ufpe.br/ revistas/revistaenfermagem/article/download/10396/11158+\&cd=1\&hl=pt-BR\&ct=clnk\&gl=br

9. Fontelles M J. Bioestatística aplicada à pesquisa experimental. São Paulo: Livraria da Física; 2012.

10. Cruz-Jentoft AJ, Baeyens JP, Bauer JM, Boirie Y, Cederholm T, Landi F, et al. Sarcopenia: European consensus on definition and diagnosis. Age Ageing [Internet]. 2010 [cited 2018 Apr 18];39(4):412-23. Available from: https://www.ncbi.nlm.nih.gov/pmc/articles/PMC2886201/ 
11. Brasil. Ministério da Saúde. Secretaria de Atenção à Saúde; Atenção à saúde da pessoa idosa e envelhecimento [Inernet]. Brasília: 2010 [citado 2017 jul. 29]. Disponível em: http://bvsms.saude.gov.br/bvs/publicacoes/abcad19.pdf

12. Fiel JNA, Lima JS, Dias JM, Neves LMT. Avaliação do risco de quedas e sarcopenia em idosos com doença pulmonar obstrutiva crônica atendidos em um hospital universitário de Belém, Estado do Pará, Brasil. Rev Pan Amaz Saúde [Internet]. 2016 [citado 2019 out. 17]; 7(4):41-5. Disponível em: http://scielo.iec.gov.br/scielo.php?script=sci_arttext\&pid=S2176-62232016000400041\&lng=pt.

13. Brasil. Ministério da Saúde. Manual para utilização da caderneta de saúde da Pessoa Idosa [Internet]. Brasília: 2018 [citado 2018 ago. 18]. Disponível em: http://bvsms.saude.gov.br/bvs/publicacoes/manual_utilizacao_caderneta_pessoa_idosa.pdf

14. Vieira S. Introdução a estatística. Rio de Janeiro: Elsevier; 2010.

15. Azevedo LM, Oliveira KMV, Nunes VMA, Alchieri JC. Perdas da capacidade funcional em idosos institucionalizados no município de Natal, Rio Grande do Norte. Rev Online Pesqui Cuid Fundam [Internet]. 2014 [cited 2018 jun. 17];6(2):485-92. Available from: http://www.seer.unirio.br/index.php/cuidadofundamental/article/view/3009/pdf_1229

16. Pinheiro NCG, Holanda VCD, Melo LA, Medeiros AKB, Lima KC. Desigualdade no perfil dos idosos institucionalizados na cidade de Natal, Brasil. Ciênc Saúde Coletiva [Internet]. 2016 [citado 2018 jul. 15]; 21(11):3399-405. Disponível em: http://www.scielo.br/scielo. php?script=sci_arttext\&pid=S1413-81232016001103399\&lng=en\&nrm=iso.

17. Camarano AA, Barbosa P. In: Alcântara AO, Camarano AA, Giacomin KC, organizadores. Política Nacional do Idoso: velhas e novas questões. Rio de Janeiro: Ipea; 2016. p. 479-511.

18. Almeida AV, Mafra SCT, Silva EP, Kanso S. A Feminização da velhice: em foco as características socioeconômicas, pessoais e familiares das idosas e o risco social. Textos Contextos (Porto Alegre) [Internet]. 2015 [citado 2018 ago. 23];14(1):115-31. Disponível em: http://revistaseletronicas.pucrs.br/ojs/index.php/fass/article/viewFile/19830/13313.

19. Souza RCF, Inacio AN. Entre os muros do abrigo: compreensões do processo de institucionalização em idosos abrigados. Pesqui Prát Psicossociais [Internet]. 2017 [citado 2018 ago. 06]; 12(1):209-223. Disponível em: http://pepsic.bvsalud.org/pdf/ppp/v12n1/15.pdf

20. Fluetti MT, Fhon JRS, Oliveira AP, Chiquito LMO, Marques S. The frailty syndrome in institutionalized elderly persons. Rev Bras Geriatr Gerontol [Internet]. 2018 Feb [cited 2018 July 22]; 21(1):60-69. Available from: http://www.scielo.br/scielo.php?script=sci_arttext\&pid $=$ S1809-98232018000100060

21. Abreu DROM, Azevedo RCS, Silva AMC, Reiners AAO, Abreu HCA. Fatores associados à recorrência de quedas em uma coorte de idosos. Ciênc Saúde Coletiva [Internet]. 2016 nov. [citado 2018 jul. 16]; 21(11):3439-3446. Disponível em: http://www.scielo.br/scielo. php?script=sci_abstract $\&$ pid=S1413-81232016001103439\&Ing=pt\&nrm=iso

22. Wu IC, Lin CC, Hsiung CA, Wang CY, Wu CH, Chan DC, et al. Epidemiology of sarcopenia among community-dwelling older adults in Taiwan: a pooled analysis for a broader adoption of sarcopenia assessments. Geriatr Gerontol Int. 2014;14 Suppl 1:52-60.

23. Ferreira LMBM, Jerez-Roig J, Andrade FLJP, Oliveira NPD, Araújo JRT, Lima KC. Prevalence of falls and evaluation of mobility among institutionalized elderly persons. Rev Bras Geriatr Gerontol [Internet]. 2016 [cited 2018 Aug 30];19(6):995-1003. Available from: http://www.scielo.br/pdf/rbgg/v19n6/pt_1809-9823-rbgg-19-06-00995.pdf

24. Santos SSC, Pelzer MT, Barros EJL, organizadoras. Quedas em idosos: reflexões a partir de produções científicas da FURG [Internet]. Rio Grande: FURG; 2010 [citado 2019 abr. 10]. Disponível em: https://eenf.furg.br/images/stories/docs/quedas.pdf

25. Olsson Moller U, Midlov P, Kristensson J, Ekdahl C, Berglund J, Jakobsson U. Prevalence and predictors of falls and dizziness in people younger and older than 80 years of age- A longitudinal cohort study. Arch Gerontol Geriatr. 2013;56(1):160-8.

26. Araújo Neto AH, Patrício ACFA, Ferreira MAM, Rodrigues BFL, Santos TD, Rodrigues TDB, et al. Falls in institutionalized older adults: risks, consequences and antecedents. Rev Bras Enferm [Internet]. 2017 [cited 2019 May 20]; 70(4):719-25. Available from: http://www.scielo. br/pdf/reben/v70n4/pt_0034-7167-reben-70-04-0719.pdf

27. Gasparotto LPR, Falsarella GR, Coimbra AMV. As quedas no cenário da velhice: conceitos básicos e atualidades da pesquisa em saúde. Rev Bras Geriatr Gerontol [Internet]. 2014 [citado 2017 jul 10]; 17(1):201-209. Disponível em: http://www.scielo.br/pdf/rbgg/v17n1/18099823-rbgg-17-01-00201.pdf

28. Ferreira LL, Sanches GGA, Marcondes LP, Saad PCB. Risco de queda em idosos institucionalizados com doença de Alzheimer. Kairós Gerontol [Internet]. 2013 [citado 2018 ago. 12];16(3). Disponível em: https://revistas.pucsp.br/kairos/article/view/18638

29. Reis LA, Rocha TS, Duarte SFP. Quedas: Riscos e fatores associados em idosos institucionalizados. Rev Baiana Enferm [Internet]. 2014 [citado 2018 ago. 28];28(3):225-34. Disponível em: https://portalseer.ufba.br/index.php/enfermagem/article/viewFile/12303/8982

30. Prata MG, Scheicher ME. Correlation between balance and the level of functional independence among elderly people. São Paulo Med J. $2012 ; 130(2): 97-101$.

Apoio financeiro

Coordenação de Aperfeiçoamento de Pessoal de Nível Superior - (CAPES). Código de Financiamento 001. 